\title{
Non-invasive monitoring of arterial wall impedance
}

\author{
Akira Sakane $^{1}$, Kenji Shiba ${ }^{1}$, Toshio Tsuji ${ }^{1}$, Noboru Saeki ${ }^{2}$, Masashi Kawamoto ${ }^{2}$ \\ ${ }^{1}$ Department of Artificial Complex Systems Engineering, Hiroshima University, Japan \\ ${ }^{2}$ Department of Anesthesiology and Critical Care, Hiroshima University, Japan
}

\begin{abstract}
This paper proposes a noninvasive technique to estimate dynamic characteristics of arterial wall by using mechanical impedance. For extracting the vascular characteristics, we use biological signals such as an electrocardiogram, noninvasive arterial pressure, and pulse oximetric plethysmogram which can be measured non-invasively. For estimating the impedance parameters with a high degree of accuracy, we define a shift time that is determined as the time difference between noninvasive arterial pressure and the plethysmogram to compensate the undesirable time delay included in the noninvasive arterial pressure. Then, the proposed method is applied to surgical operations, and the validity of the proposed method is ascertained because the estimated impedance parameters show similar changes to the ones estimated by the invasive method.
\end{abstract}

Keywords - Noninvasive monitoring, vascular system, arterial wall, plethysmogram, mechanical impedance.

\section{INTRODUCTION}

The vascular system transports various substances necessary for life, such as oxygen and nutrients, through arteries that show functional changes such as constricting and dilating with various kinds of stimuli. Also, the arteries respond to organic changes such as hardening and softening according to aging and physical conditions. Therefore, if dynamic characteristics of arteries are measured quantitatively, it is possible to estimate interior conditions of the body during surgical operations and physical training.

Many researchers have tried to reveal the dynamic characteristics of blood vessels. For example, Sawada et al. analyzed the relationship between the vascular tone and mental stress by estimating the vascular stiffness of the peripheral blood vessels [1]. Also, Katayama et al. estimated the compliance of the arterial wall with a plethysmogram and arterial pressure measurements, and other similar studies have also been reported [2],[3]. These studies, however, dealt with only stiffness or compliance, and did not sufficiently analyze dynamic vascular characteristics.

The arterial wall includes smooth muscles, whose dynamic characteristics have been analyzed in detail. Barra et al. measured time series of blood pressure and diameter of the brachial artery in conscious dogs, and calculated the stress-strain relations during vasoconstriction and vasodilation using a modified Maxwell model [4]. They also explained that the stiffness and viscosity of smooth muscles showed similar changes according to the degree of vascular contraction [5],[6]. Meanwhile, the authors modeled the dynamic characteristics of the human arterial wall by using mechanical impedance including stiffness, viscosity, and inertia, and developed a new technique to estimate the vascular impedance parameters in a beat-to-beat manner [7],[8]. The method, however, was invasive because it was necessary to insert a catheter to measure the arterial pressure.

In this paper, for estimating the vascular dynamic characteristics non-invasively, we propose a new method to estimate the vascular conditions beat-to-beat by measuring the arterial pressure non-invasively. In the proposed method, the impedance parameters can be estimated from electrocardiograms, arterial pressure, and plethysmograms with the linear least-squares method. Finally, we monitor vascular conditions using arterial wall impedance during surgical operations. The usefulness of the proposed method is confirmed comparing to the estimated results by the invasive method.

\section{IMPEDANCE Model of the ARTERIAL WALL}

\section{A. Arterial Wall Impedance}

For extracting vascular features including biological signals, we estimated the arterial wall impedance [8]. Fig.1 illustrates the proposed impedance model of the arterial wall. This model represents only the characteristics of the arterial wall in the arbitrary radius direction. The impedance characteristic can be described using stress and strain of the arterial wall as follows:

$$
d \sigma(t)=M d \ddot{\varepsilon}(t)+B d \dot{\varepsilon}(t)+K d \varepsilon(t)
$$

where $d \sigma(t)=\sigma(t)-\sigma\left(t_{0}\right) ; d \varepsilon(t)=\varepsilon(t)-\varepsilon\left(t_{0}\right) ; \sigma(t)$ is the stress exerted on the arterial wall by arterial pressure; $M, B$, and $K$ are the inertia, viscosity, and stiffness; $\varepsilon(t), \dot{\varepsilon}(t)$, and $\ddot{\varepsilon}(t)$ are the strain, strain velocity, and strain acceleration of the arterial wall. $t_{0}$ denotes the time when the $\mathrm{R}$ wave appeared in each ECG recognition cycle.

To estimate the impedance parameters given in (1), it is necessary to measure $\sigma(t)$ and $\varepsilon(t)$. The stress exerted on the tunica intima of the arterial wall is equal to arterial pressure. Then, the stress can be given as follows:

$$
\sigma(t)=P_{b}(t)
$$

where $P_{b}(t)$ is the invasive arterial pressure. On the other hand, the strain of the arterial wall $\varepsilon(t)$ is difficult to measure directly and non-invasively. Therefore, a plethysmogram is used instead of $\varepsilon(t)$. 


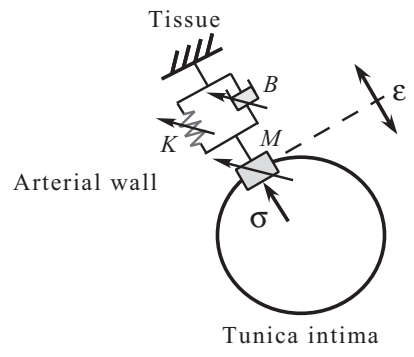

Fig. 1. Schematic description of the arterial wall impedance model

\section{B. Plethysmogram}

The variation in blood flow due to pulsations of the heart is transmitted peripherally in the arteries and fluctuates the volume of the peripheral parts. The volume changes can be measured as plethsymogram. The heart repeats contraction and dilation rhythmically, and the plethysmogram shows periodical changes with the pulsation. Therefore, we focus only on one period of the plethysmogram.

In Fig.2(a), let $D_{0}$ represent the mean value of the diameter of blood vessel $D(t)$ in a period. Also, let $I_{0}$ and $I_{D}$ denote the intensity of incident light on a blood vessel with diameter $D_{0}$ and the intensity of transmitted light, respectively, as shown in Fig. 2(a). According to LambertBeer's law [9], the following equation can be obtained:

$$
A_{0} \equiv \log \left(I_{0} / I_{D}\right)=E C D_{0}
$$

where $A_{0}$ is the absorbance proportional to the concentration of the absorptive substance $C$ and the diameter of a blood vessel $D_{0} . E$ is the specific absorption coefficient depending on the material [10]. When the diameter of the blood vessel $D(t)$ becomes larger $D_{0}+\Delta D(t)$, then the light transmitted through the blood vessel becomes $I_{D}-\Delta I(t)$. Therefore, the variation in absorbance, $\Delta A(t)$, can be given by (Fig.2(b)):

$$
\begin{aligned}
\Delta A(t) & =A(t)-A_{0} \\
& =\log \left(I_{D} /\left(I_{D}-\Delta I(t)\right)\right)=E C \Delta D(t)
\end{aligned}
$$

The variation in absorbance, $\Delta A(t)$, is defined as the plethysmogram $P_{l}(t)$. In this paper, it is assumed that $A(t)$ is proportional to the diameter of the arterial wall:

$$
A(t)=k_{p} D(t)
$$

where $k_{p}$ is the constant. Consequently, the strain of the arterial wall in the radius direction is represented as follows:

$$
\varepsilon(t)=\frac{D(t)-D_{0}}{D_{0}}=\frac{A(t)-A_{0}}{A_{0}}=\frac{P_{l}(t)}{A_{0}}
$$

\section{Arterial Wall Impedance Model}

The stress exerted on the arterial wall $\sigma(t)$ is expressed by the invasive arterial pressure $P_{b}(t)$, given by (2), and the strain of the arterial wall $\varepsilon(t)$ is represented by the plethysmogram $P_{l}(t)$ in (6). Then, the arterial wall impedance is estimated by using $P_{b}(t)$ and $P_{l}(t)$ as follows:

$$
d P_{b}(t)=\tilde{M} d \ddot{P}_{l}(t)+\tilde{B} d \dot{P}_{l}(t)+\tilde{K} d P_{l}(t)
$$

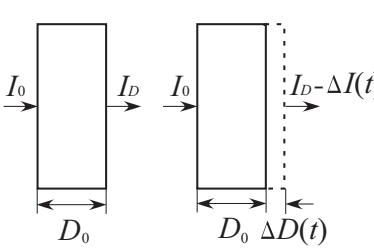

(a) Lambert-Beer's law

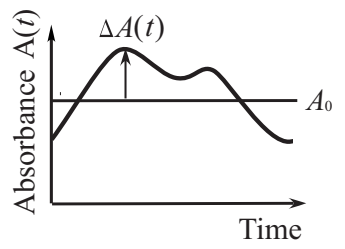

(b) Plethysmogram
Fig. 2. Theory of plethysmogram

where $d P_{b}(t)=P_{b}(t)-P_{b}\left(t_{0}\right) ; d P_{l}(t)=P_{l}(t)-P_{l}\left(t_{0}\right)$; $d \dot{P}_{l}(t)=\dot{P}_{l}(t)-\dot{P}_{l}\left(t_{0}\right) ; d \ddot{P}_{l}(t)=\ddot{P}_{l}(t)-\ddot{P}_{l}\left(t_{0}\right)$. The impedance parameters included in (7) are then given by:

$$
\tilde{M}=\frac{M}{A_{0}}, \tilde{B}=\frac{B}{A_{0}}, \tilde{K}=\frac{K}{A_{0}}
$$

where the parameter $\tilde{M}$ corresponds to the mass of the arterial wall existing in the measured part; $\tilde{B}$ and $\tilde{K}$ to the viscoelastic properties, respectively. Noting that the inertia $M$ is sufficiently small in the transversal arteries and can be ignored [11], the vascular dynamic characteristic at time $t$ can be given as follows:

$$
d P_{b}(t)=\tilde{B} d \dot{P}_{l}(t)+\tilde{K} d P_{l}(t)
$$

To estimate arterial wall impedance using the previously proposed method of [8], it was necessary to insert a catheter to measure the arterial pressure. In this paper, the noninvasive arterial pressure measured by Finapres (Finapres 2300, Ohmeda Corp.) is used to evaluate the dynamic characteristics of the arterial wall.

The Finapres measures arterial pressure non-invasively by means of a technique described by J.Penaz [12]. However, the measured noninvasive arterial pressure is delayed compared with the invasive pressure, and the delay time affects the estimated impedance parameters. Therefore, the delay time of noninvasive arterial pressure must be compensated to estimate the impedance parameters with a high degree of accuracy.

\section{Compensation of the Time Delay}

The method proposed in this paper is explained using Fig.3. The solid lines and dotted lines, respectively, indicate the measured noninvasive arterial pressure and plethysmogram. At first, the time difference of the inflection points between the measured noninvasive arterial pressure and plethysmogram is calculated, where the inflection points are determined from the leading curves to the peaks of the waves. The difference is defined as $T_{s}$ (Fig.3(a)) and used to modify the time difference between the invasive arterial pressure and plethysmogram by leading the plethysmogram $T_{s}$ (Fig.3(b)). Herewith, the system can compensate the variable time delay beat-to-beat.

Next, in order to compensate the constant delay included in the measurements, a shift time $T_{d}$ is defined and used to lead the noninvasive arterial pressure (Fig.3(b)). Consequently, the impedance parameters are estimated by using 


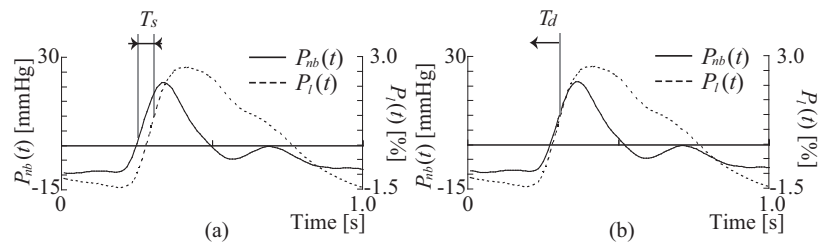

Fig. 3. The definition of shift time

the shifted invasive arterial pressure and plethysmogram. The equation for estimating impedance parameters can be expressed as follows:

$$
d P_{n b}\left(t+T_{d}\right)=\tilde{B} d \dot{P}_{l}\left(t+T_{s}\right)+\tilde{K} d P_{l}\left(t+T_{s}\right)
$$

where $d P_{n b}\left(t+T_{d}\right)=P_{n b}\left(t+T_{d}\right)-P_{n b}\left(t_{0}+T_{d}\right) ; d P_{l}(t+$ $\left.T_{s}\right)=P_{l}\left(t+T_{s}\right)-P_{l}\left(t_{0}+T_{s}\right)$; and $P_{n b}(t)$ is the noninvasive arterial pressure. The optimum shift time is determined by using the following cost function.

$$
\begin{gathered}
E_{\mathrm{a}}\left(T_{d}\right)=\frac{1}{m} \sum_{p=1}^{m} E\left(T_{d}, p\right) \\
E\left(T_{d}, p\right)=\frac{E_{\tilde{B}}\left(T_{d}, p\right)}{E_{\tilde{B}_{\max }}(p)}+\frac{E_{\tilde{K}}\left(T_{d}, p\right)}{E_{\tilde{K}_{\max }}(p)} \\
E_{\tilde{B}}\left(T_{d}, p\right)=\frac{1}{n} \sum_{t=1}^{n}\left(\tilde{B}_{\mathrm{i}}\left(t, T_{d}, p\right)-\tilde{B}_{\mathrm{ni}}\left(t, T_{d}, p\right)\right)^{2} \\
E_{\tilde{K}}\left(T_{d}, p\right)=\frac{1}{n} \sum_{t=1}^{n}\left(\tilde{K}_{\mathrm{i}}\left(t, T_{d}, p\right)-\tilde{K}_{\mathrm{ni}}\left(t, T_{d}, p\right)\right)^{2}
\end{gathered}
$$

where $\tilde{B}_{\mathrm{i}}$ and $\tilde{K}_{\mathrm{i}}$ are the impedance parameters estimated by using the invasive arterial pressure; $\tilde{B}_{\mathrm{ni}}$ and $\tilde{K}_{\mathrm{ni}}$ the estimated results of the proposed method; $E_{\tilde{B}}$ and $E_{\tilde{K}}$ the mean square error of viscosity and stiffness; $E_{\tilde{B}_{\max }}$ and $E_{\tilde{K}_{\max }}$ the maximum of $E_{\tilde{B}}$ and $E_{\tilde{K}}$ when the shift time $T_{d}$ is changed from 0 to $72 \mathrm{msec} ; m$ the number of subjects; and $n$ the number of estimated impedance parameters. When the cost function $E_{a}$ of (11) is at a minimum, we conclude it as the optimum shift time.

\section{IMPEDANCE ESTIMATION EXPERIMENTS}

\section{A. Experimental Method}

Fig.4 illustrates the experimental apparatus. An electrocardiogram $(E C G(t))$, invasive arterial pressure $\left(P_{b}(t)\right)$, noninvasive arterial pressure $\left(P_{n b}(t)\right)$, and plethysmogram $\left(P_{l}(t)\right)$ were measured simultaneously at the sampling frequency of $125 \mathrm{~Hz}$. The invasive arterial pressure was measured through a 24 gauge catheter placed in the left radial artery, and the plethysmogram was measured with the ipsilateral thumb (BSS-9800, Nihon Kohden Corp.). Also, noninvasive arterial pressure was measured at the ipsilateral middle finger (Finapres 2300, Ohmeda Corp.).

Fig.5 illustrates an example of the measured biological signals measured from a patient undergoing transsphenoidal surgery (patient A). This figure plots the electrocardiogram, arterial pressure, and plethysmogram. Because the data

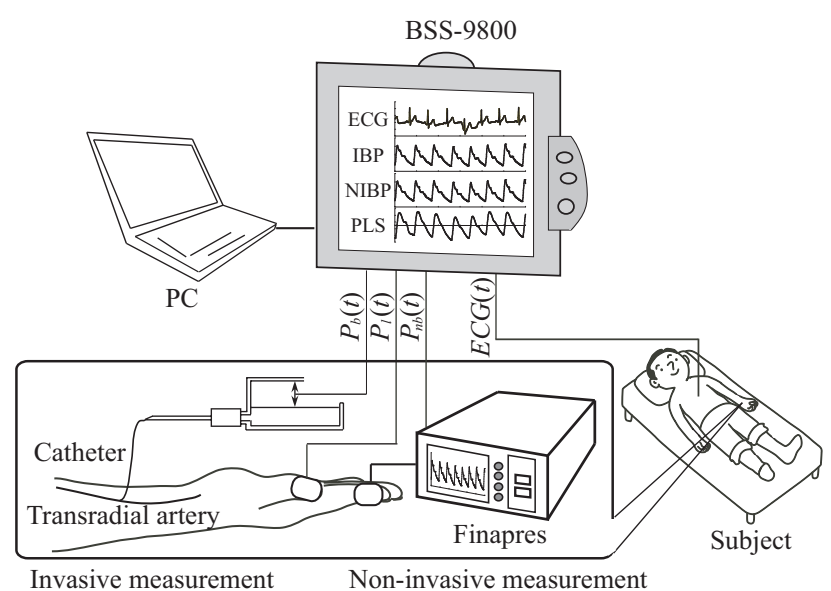

Fig. 4. Experimental instruments

were affected by some artifacts, such as light and mechanical stimulation on the patient's hand, the arterial pressure and plethysmogram were preprocessed by using digital filters. The invasive and noninvasive arterial pressures were filtered out through a second-order infinite impulse response (IIR) low-pass filter with the cutoff frequency of $6 \mathrm{~Hz}$ and a first-order IIR high-pass filter with the cutoff frequency of $0.3 \mathrm{~Hz}$, respectively, and the plethysmogram was filtered through an eighth-order finite impulse response (FIR) lowpass filter with the cutoff frequency of $15 \mathrm{~Hz}$ and a firstorder IIR high-pass filter with the cutoff frequency of 0.3 $\mathrm{Hz}$. The $\mathrm{R}$ wave generally had distinctly large amplitude and so was used in this study to mark ECG signal divisions. Defining the time when the $\mathrm{R}$ wave appeared in each ECG recognition cycle as $t_{0}, d P_{b}(t), d P_{n b}\left(t+T_{d}\right), d P_{l}(t)$, $d \dot{P}_{l}(t), d P_{l}\left(t+T_{s}\right)$, and $d \dot{P}_{l}\left(t+T_{s}\right)$ for the interval between the $\mathrm{R}$ wave and the subsequent $\mathrm{R}$ wave (RR interval) were used to determine the impedance parameters of (9) and (10). Because the previous RR interval was established each time an $\mathrm{R}$ wave was detected, the beat-to-beat arterial wall impedance parameters $\tilde{B}$ and $\tilde{K}$ could be estimated [8].

\section{B. Estimation Results}

The evaluation of the shift time was performed by using the biological signals measured from subjects who underwent surgeries $(m=4)$. The data were collected in a relatively relaxed condition during surgeries, and estimated impedance parameters $(n=100)$ were used to calculate the cost function $E$ of (12). Fig.6 shows the calculated results of the cost function $E$, where the sampling frequency was $125 \mathrm{~Hz}$. These results show that the cost function $E$ reaches a minimum when the shift time $T_{d}$ is $32 \mathrm{msec}$ or 42 msec for each subject, and it was found that the individual difference was not so much in the measured data. Then, the average cost function $E_{a}$ of (11) was calculated. Fig.7 shows the result of the average cost function $E_{a}$. From this result, the shift time $T_{d}=32 \mathrm{msec}$ was determined as the optimum shift time.

Then, we estimated the impedance parameters by using 


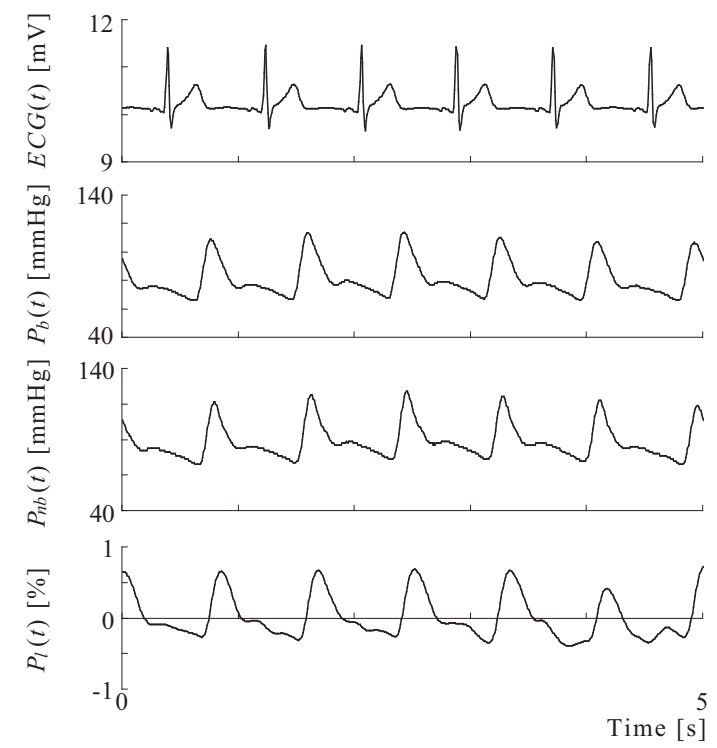

Fig. 5. Examples of the biological signals measured from the patient A

the shift time $T_{d}=32$ msec. Fig. 8 shows an example of the plethysmogram and noninvasive arterial pressure measured from patient A, where the figures (a), (b), and (c) represent the time courses of the variation in the plethysmogram $d P_{l}\left(t+T_{s}\right)$, the first derivative of the plethysmogram $d \dot{P}_{l}\left(t+T_{s}\right)$, and the variation in noninvasive arterial pressure $d P_{n b}\left(t+T_{d}\right)$, respectively. The solid line and the dotted line, respectively, indicate the measured pressure and the estimated pressure obtained by substituting the estimated impedance parameters, $\tilde{B}$ and $\tilde{K}$, and the measured plethysmogram to (10). Fig. 8 shows that the vascular characteristics are well expressed with the proposed model in (10) because good agreement between measured and estimated pressures is obtained. From this result, it was found that compensation of the time delay is useful to evaluate the dynamic characteristics of arterial wall noninvasively.

\section{Impedance Monitoring of the ARterial WALL}

The proposed method has the potential to be used as a monitoring system for vascular conditions during surgery. We therefore attempted to monitor changes in arterial wall impedance during surgery.

\section{A. Impedance Monitoring}

The proposed method was used to monitor the impedance of the arterial wall during transsphenoidal surgery (patient A) and endoscopic thoracic sympathectomy (patient B). The transsphenoidal surgery is one of the most typical keyhole surgeries, where the surgeons approach the pituitary tumor behind sphenoid through the narrow nasal cavity to remove the tumor [13]. Also, the endoscopic thoracic sympathectomy is a surgery for hyperhidrosis patient. The sympathetic nerves of hyperhidrosis patient are hyperfunction of sympathetic nerve, so that the patient perspire copiously in the
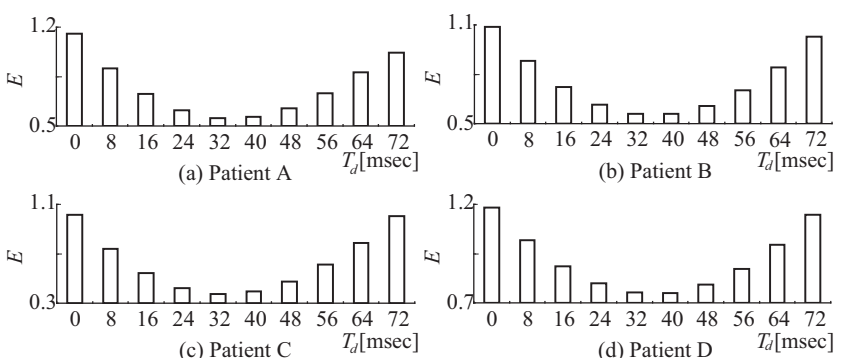

Fig. 6. The cost function $E$ with the shift time $T_{d}$

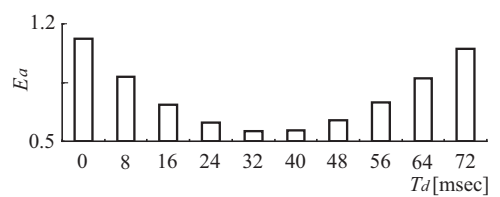

Fig. 7. The cost function $E_{a}$ with the shift time $T_{d}$

palms and armpits compared to ordinary people. In this operation, the sympathetic nerve running along the spine is clipped to stop perspiration [14].

The estimated impedance are shown in Figs.9 and 10. The results represent the viscosity, stiffness, and the coefficient of determination, respectively. The value of $R^{2}$ varies from zero to one, and small $R^{2}$ means that the goodness of fit is low. In this paper, the estimated results in which the coefficient of determination was greater than 0.8 were accepted as the estimated impedance.

Fig.9 shows the estimated results of patient A. The patient underwent surgery accompanied by severe pain during 1800-2500 sec, and blood vessels constricted in the shaded area. Strong stimuli were not added after that, and the estimated values were also stable relatively. During the latter part of the operation (after the dotted line of Fig.9), the effect of anesthesia was wearing off and the stiffness increased slowly.

Fig. 10 shows the estimated results of patient B, where the sympathetic nerve of the patient was clipped at the shaded area. The surgeon stimulated the patient's tissues to identify the sympathetic nerve before it was clipped. When these painful stimuli were applied, sensory information was transmitted from the medulla spinalis to the brain, and peripheral vasoconstriction and muscle contraction occurred. Therefore, peripheral vascular tone (elasticity) increased. After the sympathetic nerve was clipped, the blood vessels became markedly compliant because sympathetic nerve signals could not be transmitted to the peripheral part [14]. During the latter part of the operation (after the dotted line of Fig.10), the patient felt pain because the effect of anesthesia was wearing off. The impedance parameters of the noninvasive method were not estimated at 2800$3000 \mathrm{sec}$ because the Finapres was stopped to prevent any complications. Our results show that the estimated impedance parameters corresponded well with the surgical events. From these results, since the estimated values of the proposed method showed similar changes to those of the invasive method, the usefulness of proposed method was 


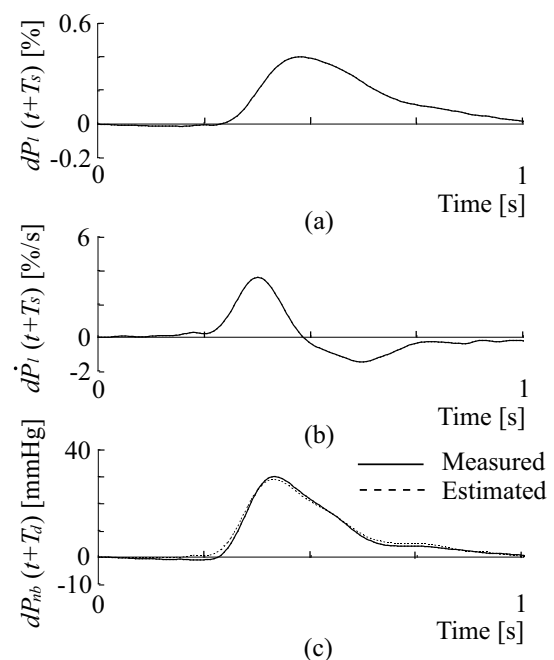

Fig. 8. Example of the plethysmogram and the non-invasive arterial pressure

ascertained.

\section{B. Differences between Invasive and Noninvasive Arterial Pressures}

It can be seen from Fig.10 that the values of impedance parameters estimated by using the proposed method is considerably different with the parameters estimated by using the invasive method, especially, just before the sympathetic block. Fig.11 shows the enlarged portion of estimated impedance parameters at 3300-4200 sec. The shaded area of Fig.11(b) (3670-3890 sec) shows the estimation results when the blood vessels were extremely constricted. This indicates that there is substantial margin of error between the invasive and noninvasive estimation results. The example of the measured arterial pressure at this timing is shown in Fig.12(a). The solid line and the dotted line, respectively, indicate the invasive pressure and the noninvasive pressure. From this result, the noninvasive arterial pressure is attenuated if compared to the invasive arterial pressure. It is considered that the attenuation is caused by inadequate blood flow volume to the peripheral part with vasoconstriction. Then, we tried to modify the gap between noninvasive and invasive arterial pressures using the system identification technique for clarifying the transfer characteristic of arterial pressure in the peripheral part. [15]:

An autoregressive model with exogenous input is used

$$
\begin{array}{r}
y(t)=-a_{1} y(t-1)-\cdots-a_{n a} y\left(t-n_{a}\right) \\
+b_{1} u(t-k)+\cdots+b_{n b} u\left(t-k-n_{b}+1\right)+\omega(t)
\end{array}
$$

where $u(t)$ and $y(t)$ are the noninvasive and invasive arterial pressures, respectively; $t$ denotes discrete time; $k$ is the time delay between the input $u(t)$ and the output $y(t) ; \omega(t)$ is the residual error between the actual and model-estimated outputs; and $n_{a}$ and $n_{b}$ represent the number of $a$ and $b$
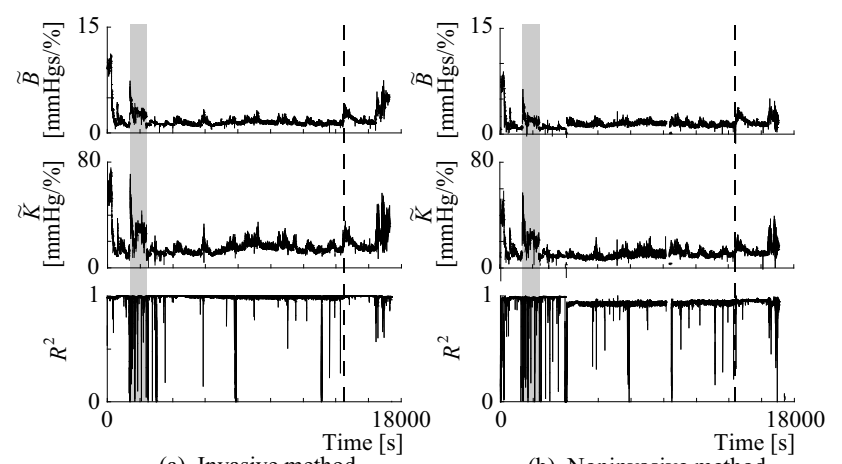

(a) Invasive method

(b) Noninvasive method

Fig. 9. Impedance monitoring during transsphenoidal surgery (Patient A)

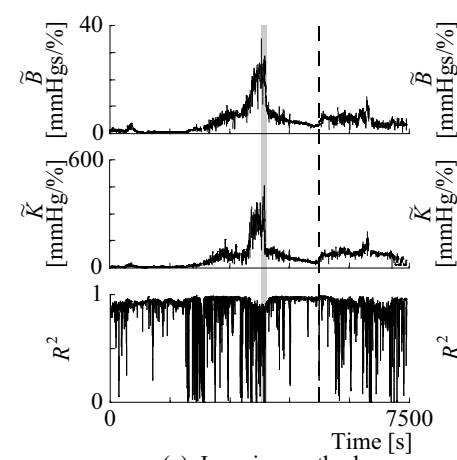

(a) Invasive method

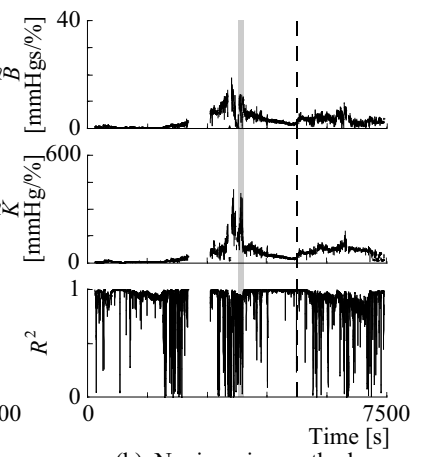

(b) Noninvasive method

Fig. 10. Impedance monitoring during endoscopic thoracic sympathectomy (Patient B)

coefficients of the model. The order of the ARX model was determined from Akaike information criterion [15]. After the transfer characteristic between noninvasive and invasive arterial pressures was clarified, we modified the measured noninvasive arterial pressure by applying the ARX model to estimate the invasive arterial pressure.

Then, the attenuation ratio $D_{\text {ratio }}$ was calculated as follows:

$$
D_{\text {ratio }}=\frac{P_{n b_{\max }}-P_{n b_{\min }}}{P_{b_{\max }}-P_{b_{\min }}}
$$

where $P_{n b_{\max }}$ and $P_{n b_{\min }}$ are the maximum and minimum values of noninvasive arterial pressure during each beatto-beat interval, respectively; and $P_{b_{\max }}$ and $P_{b_{\min }}$ the corresponding ones of the invasive arterial pressure. The identification of ARX model was performed separately depending on the attenuation ratio: $0.5<D_{\text {ratio }} \leq 0.8$ and $D_{\text {ratio }} \leq 0.5$. Also, the modification was not executed when the attenuation ration is $D_{\text {ratio }}>0.8$.

The modified arterial pressure is described in Fig.12(b). The solid line and the dotted line, respectively, indicate the measured and the modified arterial pressure. It can be seen that the measured and modified pressures agreed well. Then, the impedance parameters estimated by using the modified arterial pressure are shown in Fig.11(c). The faint shaded areas correspond to when the attenuation ratio is 0.5 $<D_{\text {ratio }} \leq 0.8$, and the dark shaded area corresponds to $D_{\text {ratio }} \leq 0.5$. To find out how the estimation accuracy was 

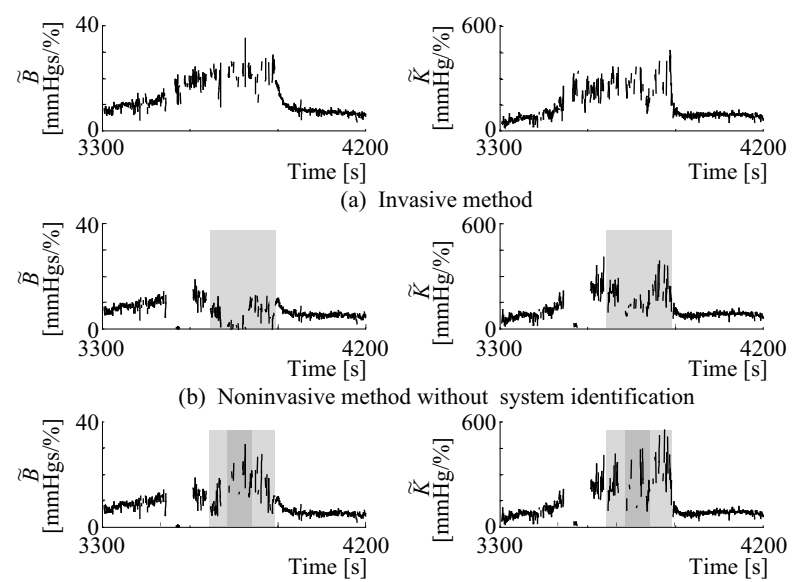

(c) Noninvasive method with system identification

Fig. 11. The estimated results during endoscopic thoracic sympathectomy
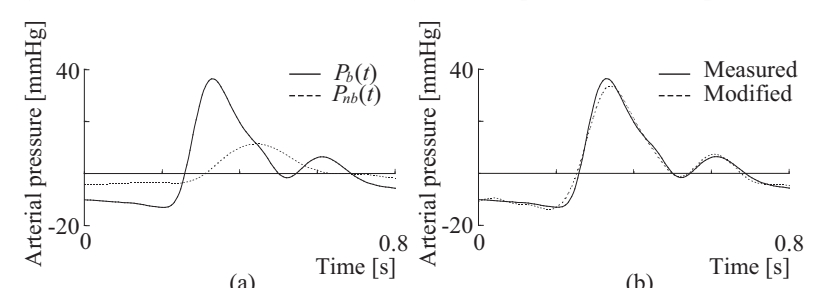

Fig. 12. Measured and modified arterial pressures

improved by the system identification, we calculated $E_{\tilde{K}_{a}}$ and $E_{\tilde{B}_{a}}$ at the shaded area as follows:

$$
\begin{aligned}
& E_{\tilde{K}_{a}}=\frac{1}{n} \sum_{t=1}^{n}\left|\left(\tilde{K}_{i}(t)-\tilde{K}_{n i}(t)\right)\right| \\
& E_{\tilde{B}_{a}}=\frac{1}{n} \sum_{t=1}^{n}\left|\left(\tilde{B}_{i}(t)-\tilde{B}_{n i}(t)\right)\right|
\end{aligned}
$$

where $n$ is the number of estimated impedance parameters at the shaded area. As a result, $E_{\tilde{K}_{a}}$ was $93.32 \mathrm{mmHg} / \%$ and $E_{\tilde{B}_{a}}$ was $15.34 \mathrm{mmHgs} / \%$ before the system identification, and $E_{\tilde{K}_{a}}$ was $34.88 \mathrm{mmHg} / \%$ and $E_{\tilde{B}_{a}}$ was $4.42 \mathrm{mmHgs} / \%$ after the system identification. From these results, the error was reduced to about one-third, and the dynamic characteristics of blood vessels may be possible to estimate with high accuracy, even when the blood vessels were extremely constricted.

\section{CONCLUSiON}

We have proposed a noninvasive estimation method of arterial wall impedance. For estimating the impedance parameters with high accuracy, we defined the shift time and determined the optimal shift time through experiments. The proposed method was then used to monitor the arterial wall impedance during surgery. The experiments revealed that the changes in impedance corresponded with surgical events. Also, the estimated impedance parameters changed similar to those of the invasive method. Finally, it was ascertained that the impedance parameters were estimated with high accuracy by using the system identification technique when the blood vessels were extremely constricted.
Future research will be directed to test weather the proposed model can use with other subjects. Also, we will try to develop a practical system for estimating the vascular conditions non-invasively and ascertain the usefulness of the proposed method in general environments.

\section{ACKNOWLEDGEMENT}

The authors would like to thank T.Ukawa and K.Tone of the NIHON KOHDEN Corp. for making available clinical data and for their precious suggestions.

This work was supported by Grant-in-Aid for Scientific Research of Japan Society for the Promotion of Science (1508279) and Research Fund of Hiroshima Prefectural Institute of Industrial Science and Technology.

\section{REFERENCES}

[1] Y.Sawada, G.Tanaka, and K.Yamakoshi, "Normalized pulse volume derived photo-plethysmographically as a more valid measure of the finger vascular tone," Int. J. Psychophysiol., Vol. 41, No. 1, pp. 1-10, 2001.

[2] K.Katayama, M.Shimoda, J.Maeda, and T.Takemiya, "Endurance Exercise Training Increases Peripheral Vascular Response in Human Fingers," Jpn. J. Physiol., Vol. 48, No. 5, pp. 365-371, 1998.

[3] N.Saeki, M.Kawamoto, and O.Yuge, "Quantitive view of peripheral circulation," 30th International Educational and Scientific Symposium of the Society of Critical Care Medicine, Feb. 10-14, USA, 2001.

[4] J.G.Barra, et al., "Assessment of Smooth Muscle Contribution to Descending Thoracic Aortic Elastic Mechanics in Conscious Dogs," Circulation Research, Vol. 73, pp. 1040-1050, 1993.

[5] R.Armentano, et al., "Effects of Hypertension on Viscoelasticity of Carotid and Femoral Arteries in Humans," Hypertension, No. 26, pp. 48-54, 1995

[6] J.G.Barra, S.Graf, and R.L.Armentano, "Beneficial Effect of Aortic Smooth Muscle Energy Dissipation in Conscious Dogs," in Proc. World Congress on Medical Physics and Biomedical Engineering, 531, 2003.

[7] A.Sakane, T.Tsuji, Y.Tanaka, N.Saeki, and M.Kawamoto, "Estimating Arterial Wall Impedance using a Plethysmogram," in Proc. the 29th Annual Conference of the IEEE Industrial Electronics Society, November, USA, pp. 580-585, 2003.

[8] A.Sakane, T.Tsuji, Y.Tanaka, N.Saeki, and M.Kawamoto, "Monitoring of Vascular Conditions Using Plethysmogram," The Society of Instrument and Control Engineers, Vol. 40, No. 12, pp. 1236-1242, 2004 (in Japanease).

[9] R.A.Day and A.L.Underwood, QUANTITATIVE ANALYSIS 4th Edition, Prentice-Hall, 1980.

[10] T.Aoyagi and T.Ukawa, "Theory and structure of Pulseoximeter," Clinical Engineering, Vol. 7, No. 2, pp. 102-110, 1996 (in Japanese).

[11] W.W.Nichols and M.F.O'Rourke, McDonald's Blood Flow in Arteries: Theoretical, experimental and clinical principles, Edward Arnold, 1998.

[12] J.Penaz, "Photoelectric measurement of blood pressure, volume and flow in the finger," In Digest of the 10th Int. Conf. Med. Biol. Engineering, Dresden, 104, 1973.

[13] Y.Koseki, T.Washio, K.Cchinzei, and H.Iseki, "Endoscope Manipulator for Trans-nasal Neurosurgery, Optimized for and Compatible to Vertical Field Open MRI," in Proc. of MICCAI 2002, Part I, pp. 114-121, 2002.

[14] C.Drott, G.Gothberg, and G.Claes, "Endoscopic transthoracic sympathectomy: an efficient and safe method for the treatment of hyperhidrosis," Journal of the American Academy of Dermatology, Vol. 33, No. 1, pp. 78-81, 1995.

[15] L.Ljung, System Identification: Theory for the User, 1st ed. Englewood Cliffs, NJ: Prentice-Hall, 1987. 